\title{
Investigation of Fatigue Crack Propagation Behavior in Ultrafine-Grained Steel by AFM and EBSP
}

\author{
Hidehiko Kimura, Dept. of Mechanical Engineering, Nagoya Univ., Nagoya, Japan \\ Keisuke Tanaka, Dept. of Mechanical Engineering, Nagoya Univ., Nagoya, Japan \\ Yoshiaki Akiniwa, Dept. of Mechanical Engineering, Nagoya Univ., Nagoya, Japan \\ Yasuaki Tahara, Graduate School of Mechanical Engineering, Nagoya Univ., Nagoya, Japan \\ Tadashi Ishikawa, Steel Research Lab., Technical Development Bureau, Nippon Steel Corporation, Futtsu-city, Japan
}

Ultrafine-grained materials are expected to improve structural integrity due to the excellent material properties by the grain size effect without addition of special alloying elements.

Steel plates produced by an advanced thermo-mechanical control process have two regions: the ultrafine-grained surface layer with the average grain size of less than $2 \mu \mathrm{m}$ and the intermediate region with medium-sized grains. The steel plates, called SUF steel, exhibit excellent crack arrestability against brittle crack propagation. Although SUF steel has been proven to possess superior fatigue properties as well, the mechanism of the improvement in the fatigue properties is not understood.

In this study, smooth specimens and single-edge-notched specimens prepared from the ultrafine-grained and medium-grained regions were fatigued under cyclic axial tension compression at room temperature. The effect of the microstructures on the fatigue limits and crack propagation behavior was investigated. The observation of the fatigue crack initiation and early propagation behavior of small cracks were also conducted by AFM and crystallographic analysis based on EBSP.

The results show that the crack propagation rate, when compared at the same stress intensity range, was lower in the ultrafine-grained specimen. The threshold value of the stress intensity range in the ultrafine-grained specimen was higher than that in the medium-grained specimen, indicating that the ultrafine-grained specimen exhibited not only higher fatigue limits but also superior crack arrestability against long fatigue cracks. The higher crack arrestabiliy in the ultrafine-grained specimen is attributed to the bifurcation of fatigue cracks.

The investigation on the fatigue crack initiation shows that the fatigue cracks initiated from the simple slip lines in the medium-grained specimens. On the other hand, cross slips were observed near the grain boundaries prior to the initiation of fatigue cracks in the ultrafine-grained specimens. The fatigue cracks were nucleated at the grain boundary between the grains with high density of the cross slips. The crystallographic analysis clarified the mechanism of the formation of the cross slips and the subsequent crack initiation process. The crack propagation rate of the small fatigue crack in the ultrafine-grained specimens decreases because of the grain-boundary blocking and crack deflection at an early stage of the propagation. 